\title{
The spraying process simulation of the low- volume sprayer working body
}

\author{
Li Afanasiy ${ }^{1 *}$, Burkhan Utepov $^{1}$, and Satniyaz Allaniyazov ${ }^{2}$ \\ ${ }^{1}$ Tashkent Institute of Irrigation and Agricultural Mechanization Engineers, Tashkent, Uzbekistan \\ ${ }^{2}$ Branch of the Tashkent State Agrarian University in Nukus, Nukus, Uzbekistan
}

\begin{abstract}
The article presents the developed mathematical models that describe the process of spraying the working body of a low-volume sprayer. Theoretical studies show that with an increase in the supply of working fluid to the atomizing disc, the rotational speed of the pneumatic disc atomizer decreases, and in order to ensure the effect of the air flow on the droplet formation process, the radius of the disc should be larger than the radius of the base of the cone-shaped fairing, but less than its maximum critical value and for obtaining a monodisperse spray with the main droplet diameter $\mathrm{d}=80 \ldots 120 \mu \mathrm{m}$, at an axial air flow velocity of the fan installation $U=40 \ldots 60 \mathrm{~m} / \mathrm{s}$, the parameters of the disk the sprayer and the propeller are linked together when the following values: disk radius $r=65$ $\ldots 85 \mathrm{~mm}$, the number of radial channels on the disk $\mathrm{np}=2 \ldots 6 \mathrm{pcs}$, the width of the radial channel $\mathrm{bp}=3 \ldots 4 \mathrm{~mm}$, and to obtain a high-quality air-droplet flow, the initial velocity of the main drops discharged from the periphery of the spraying disc must be less than the speed of the air flow and, at the same time, the rotational speed of the pneumatic disc sprayer is recommended to be used in the range of $\omega=60 \ldots 200 \mathrm{~s}-1$.
\end{abstract}

\section{Introduction}

Cotton is the main and most valuable technical crop cultivated on the territory of the Republic of Uzbekistan [1-3].

The most labor-intensive and technically complex operation in the cultivation of cotton is harvesting, so the mandatory and most important condition for the preparation of cotton crops for productive and high-quality work of cotton harvesters is the artificial removal of cotton leaves by carrying out pre-harvest defoliation [4].

Among the many known methods of treating plants with pesticides (pollination, spraying, fumigation, aerosols, etc.), the most effective in the economic, agrotechnical and environmental aspects is spraying, through which about $75 \%$ of the work volume is performed [5]. Therefore, creating a high-performance sprayer that meets the requirements of low-volume spraying of cotton is an urgent task.

The purpose of the study is to study the process of liquid spraying by a smooth rotating disk with a coaxially lateral air flow to create a working body and a technological scheme for a low-volume method of spraying cotton.

*Corresponding author: magnaty_82@list.ru 


\section{Materials and Methods}

The analytical study of the process of spraying fluid rotating spray driven by the air flow used in the theory of rotating nozzles and wind turbines, based on which analytical dependencies that determine the optimal monodisperse droplet sizes depending on the radius of the nozzle, the specific flow of the fluid, the frequency of the rotating disk atomizer and other factors [6-13].

\section{Results and Discussion}

When studying the fluid flow inside the disk package, we assume that the thickness of the viscous fluid layer is small compared to the disk size, and the fluid flow is axisymmetric. We also assume that the path of the liquid particle is straight and the particle moves along the radius; we assume that there is no sliding of the liquid relative to the disk's surface, i.e. the gravity is significantly less than the centrifugal force.

Then the equation of motion of a liquid film is described by a system of Navew-Stokes differential equations. Still, due to the small thickness of the film, this system can be simplified and reduced to a single differential equation:

$$
\mu \frac{d^{2} U_{j}}{d Z^{2}}+\rho_{j} r \omega^{2}=\mathrm{o}
$$

Boundary conditions on:

the wall: $Z=0, U_{j}=0$

free surface area: $Z=\delta_{1}, \frac{d U_{j}}{d Z}=0$

The solution of equation (1) with boundary conditions is written as follows:

$$
U_{j}=\frac{\rho_{j} r \omega^{2}}{\mu}\left(\delta z-\frac{Z^{2}}{2}\right)
$$

The value of $\mathrm{Z}$ is determined from the condition that the total fluid flow in the layer is equal to the liquid flow rate $\mathrm{q}$, filed with the disc set.

Then:

$$
q=\int_{0}^{\delta} 2 \pi r U_{j} d z=\frac{2 \pi \rho_{j} r^{2} \omega^{2} \delta^{3}}{3 \mu}
$$

Where do we get the thickness of the liquid layer:

$$
\delta=\sqrt[3]{\frac{3 \mu q}{2 \pi \rho_{j} r^{2} \omega^{2}}}
$$

As can be seen from expression (4), the thickness of the liquid film on the disk surface is directly proportional to the flow rate of the liquid. To meet the conditions of monodisperse liquid spraying with rotating sprayers, many scientists have proved that the liquid consumption per 1 disk should not exceed 1 litre per minute [14-21]. Based on this, we can assume that the number of $n$ disks in the package should be greater than the minute flow rate q of liquid or for a package of disks:

$$
q_{i}=\frac{q}{n} \leq 1 \frac{l}{\min }
$$

Then equation (4) will take the following form: 


$$
\delta=\sqrt[3]{\frac{3 \mu q}{2 \pi \rho_{j} r^{2} \omega^{2} n}}
$$

To obtain a one-sided film cover on the surface of the disk and prevent the flow of liquid between two adjacent disks in the disk package, the distance between them should be:

$$
l_{g}=\delta
$$

Figure 1 shows the dependence of the distance $l_{g}$ between adjacent disks on the radius $r$ and the rotational speed $\omega$ at a constant liquid supply $q=$ const. As you can see from the graph, as the radius and rotation speed of the disk increase, the distance between adjacent disks $l_{g}$ decreases [22].

To determine the number of radial channels $p_{p}$ and the cross-sectional area $S_{p}$ of the radial channels, consider the condition of continuity of the fluid flow:

$$
\begin{aligned}
& q=q_{1}+q_{2}+\cdots+q_{n}+q_{n}=\sum n q_{i} \\
& S_{B} U_{j}=\mathrm{n} \sum S_{p} n_{p} U_{j}
\end{aligned}
$$

Then equation (9), after some simplifications, has the form:

$$
S_{p} n_{p}=\frac{q}{n U_{j} l_{g}}
$$

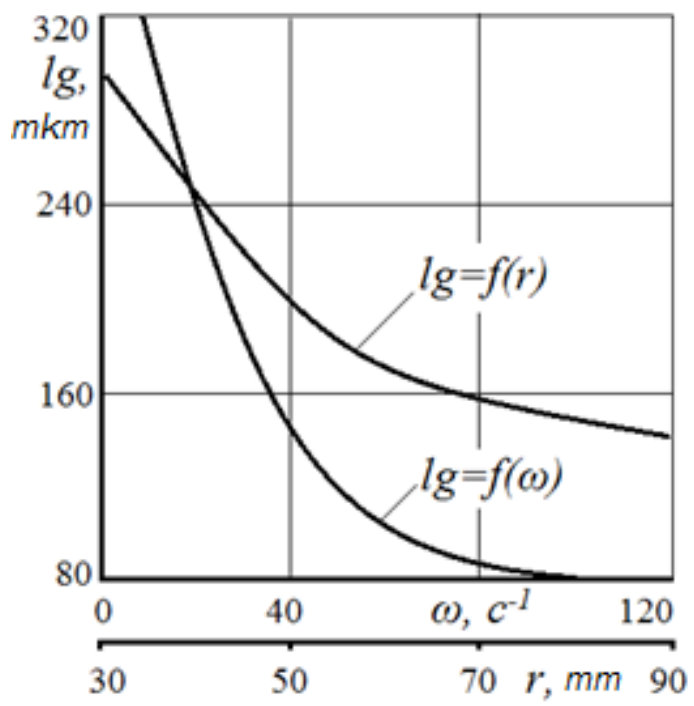

Fig. 1. Dependence of the distance $l_{g}$ between adjacent disks on $r$ and $\omega$

It follows from expression (10) that the cross-sectional area $S_{p}$ and the number $\mathrm{n}_{\mathrm{p}}$ of radial channels between adjacent disks equally affect the nature of the change in fluid motion. For $\Delta h_{p}=l_{g}$, i.e., assume that the height of the radial channel $h_{p}$ is equal to the distance $l_{g}$ between adjacent disks, and if the conditions (5) are met, the expression (10) can be written as follows:

$$
b_{p} h_{p}=\frac{q}{n U_{j} l_{g}}
$$


Figure 2 shows the dependence of the width of the radial channel $b_{p}$ on the number of radial channels $n_{p}$, for $h_{p}=l_{g}$ and $U_{j}=$ const.

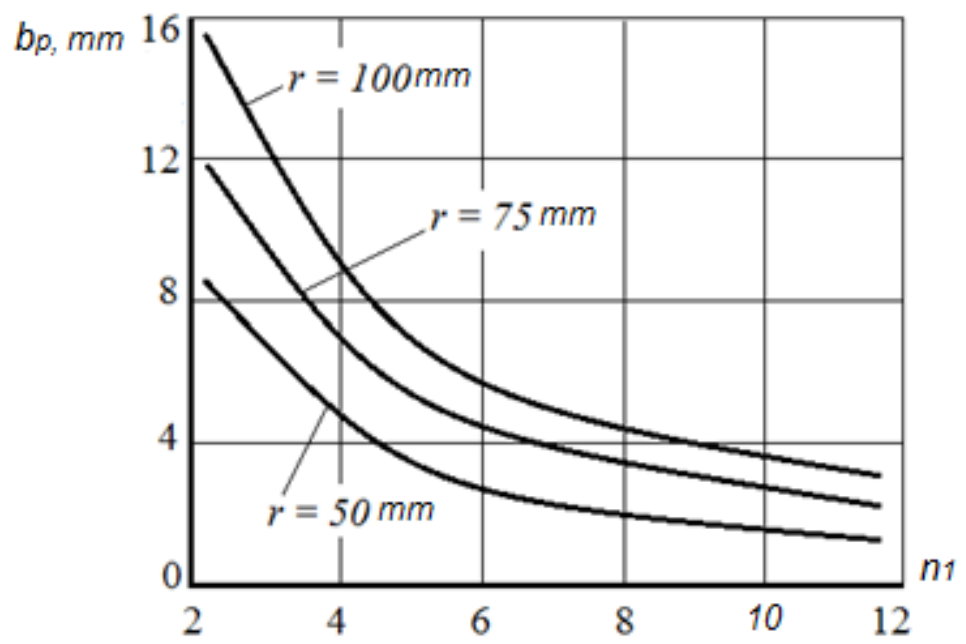

Fig. 2. Determination of the width $b_{p}$ of the radial channel depending on the number of $n_{p}$ radial channels at $h_{p}=l_{g}$ and $U_{j}=$ const

The graph shows that the number of radial channels $n_{p}$ disproportionately affects the width $b_{p}$ of the radial channel.

The speed of rotation of the pneumatic disk sprayer can be determined by the formula:

$$
\omega=2 U_{1}^{3} \sqrt[3]{\frac{\rho_{B} \xi}{m_{i}+m_{j}}}
$$

where $m_{i}$ is weight of the rotating part of the sprayer, $\mathrm{kg} ; m_{j}$ is second mass of liquid supplied to the sprayer, $\mathrm{m} / \mathrm{s}$.

The second mass of the liquid supplied to the sprayer is determined by the formula:

$$
m_{j}=q \rho_{j} t
$$

To determine the time $t$ required for the departure of the elementary mass of the liquid from the edge of the disk, consider the movement of the liquid on the surface of the disk sprayer.

For a stationary observer, the trajectory of a particle is an Archimedean spiral (Figure 3 ), the equation of which has the form:

$$
p=\mathrm{a}_{1} \varphi
$$

where $\mathrm{a}_{1}=\frac{U_{j}}{\omega}$ is constant value, $\mathrm{m} ; \varphi$ is angle from 0 to $+\infty$. 


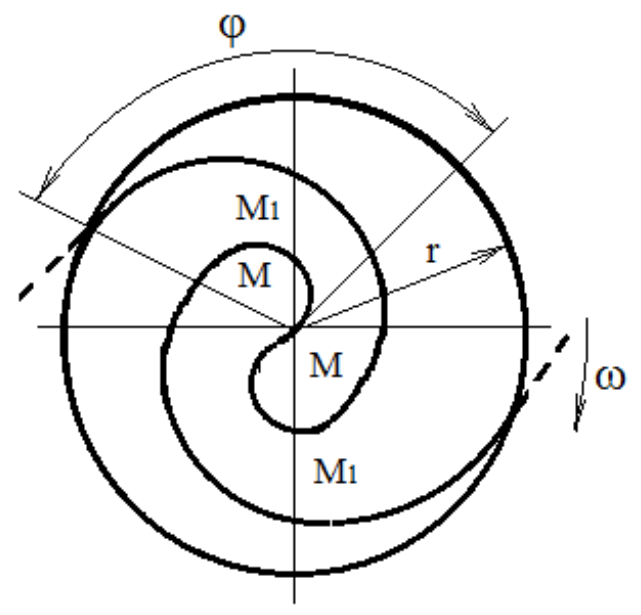

Fig. 3. Archimedes ' Spiral

The distance $\mathrm{MM}_{1}$ between adjacent turns is determined by:

$$
M M_{1}=2 \pi \mathrm{a}_{1}=2 \pi \frac{U_{j}}{\omega}
$$

Then the number of turns on the surface of the disk with radius $r$ is:

$$
\mathrm{K}_{\mathrm{B}}=\frac{r}{M M_{1}}=\frac{r \omega}{2 \pi U_{j}}
$$

From the expression (16), we find the twist angle $\varphi$ of an elementary particle of liquid on the surface of the disk:

$$
\varphi=2 \pi K_{\mathrm{B}}=\frac{r \omega}{U_{j}}
$$

The length of the arc $1_{n}$ from the center to the edge of the disk with radius $r$, is the distance travelled by the particle:

$$
l_{n}=\frac{\mathrm{a}_{1}}{2}\left[\varphi \sqrt{\varphi^{2}+1}+l_{n}\left(\varphi+\sqrt{\varphi^{2}+1}\right)\right]
$$

The time $t$ required for the movement of a liquid particle from the center to the edge of the disk is determined from the following expression:

$$
t=\frac{l_{n}}{U_{j}}=\frac{1}{2 \omega}\left[\varphi \sqrt{\varphi^{2}+1}+\left(\varphi+\sqrt{\varphi^{2}+1}\right)\right]
$$

Substituting the value $t$ in the expression (13), we find the frequency $\omega$ of rotation of the disk sprayer as a function of the air flow velocity $U$ and the specific flow rate $\mathrm{q}$ of the liquid:

$$
\omega=2 U \sqrt[3]{\frac{\rho_{\mathrm{B}} \xi}{m_{i}+\frac{\rho_{\dddot{K}} q}{2 \omega}\left(\varphi \sqrt{\varphi^{2}+1}+l_{n}\left(\varphi+\sqrt{\left.\varphi^{2}+1\right)}\right)\right.}}
$$


Converting the expression (20) will result in the following form:

$$
m_{i} \omega^{3}+\frac{\rho_{j} q}{2}\left(\varphi \sqrt{\varphi^{2}+1}+l_{n}\left(\varphi+\sqrt{\left.\varphi^{2}+1\right)}\right) \omega^{2}=8 U_{1}^{3} \rho_{\mathrm{B}} \xi\right.
$$

Provided that $\varphi \rightarrow \infty$ equation (21) takes the following form:

$$
m_{i} \omega^{3}+\frac{\rho_{\circledast} q r^{2}}{2 \mathrm{a}_{1}^{2}} \omega^{2}=8 U_{1}^{3} \rho_{\mathrm{B}} \xi
$$

Solving the cubic equation, we find the rotation frequency $\omega$ of the disk sprayer:

$$
\begin{gathered}
\omega=\left[\sqrt[3]{\frac{2 U_{1}^{3} \rho_{B} \xi}{m_{i}}-\frac{\rho_{j}^{3} q^{3} r^{6}}{\delta^{3} a_{1}^{6} m_{i}^{3}}+\sqrt{\frac{\rho_{j}^{3} q^{3} r^{2}}{\delta^{3} a_{1}^{6} m_{i}^{3}}-\frac{2^{4} U_{1}^{6} \rho_{B}^{2} \xi^{2}}{m_{i}^{2}}}}+\right. \\
\left.+\sqrt[3]{\frac{2 U_{1}^{3} \rho_{\mathrm{B}} \xi}{m_{i}}-\frac{\rho_{\varkappa}^{3} q^{3} r^{6}}{\delta^{3} a_{1}^{6} m_{i}^{3}}-\sqrt{\frac{\rho_{\kappa}^{3} q^{3} r^{2}}{\delta^{3} a_{1}^{6} m_{i}^{3}}-\frac{2^{4} U_{1}^{6} \rho_{\mathrm{B}}^{2} \xi^{2}}{m_{i}^{2}}}}\right]-\frac{\rho_{\varkappa} q r^{2}}{6 a^{3} m_{i}}
\end{gathered}
$$

\section{Conclusions}

The equation analysis shows that the increase in the supply of working fluid to the atomizing disk reduces the speed of rotation of the pneumatic disk sprayer.

Also, for the high-quality operation of the pneumatic disk sprayer, the following conditions must be met:

-to ensure the influence of the air flow on the process of droplet formation, the radius of the disk must be greater than the radius of the base of the cone-shaped fairing, but less than its greatest critical value:

$$
r_{k}<r<r_{\text {max }}
$$

-below the resulting key drops, with a drop from the periphery of the atomizer significant initial velocity, are unable to "break" the air current and go beyond it, and be carried away fully air flow speed Pavlovskogo atomizer must satisfy the inequality:

$$
\omega=\frac{U}{2 \pi r}
$$

-to obtain a monodisperse spray with major drops diameter $d=80 \ldots 120 \mu \mathrm{m}$, and the axial velocity of the air ventilation unit $U=40 \ldots 60 \mathrm{~m} / \mathrm{s}$, disk atomizer and propeller are linked with the following: the radius of the disk $r=65 \ldots 85 \mathrm{~mm}$, number of radial channels on the disc or $n_{p}=2 \ldots 6 \mathrm{PCs}$, the radial width of the channel $b_{p}=3 \ldots 4 \mathrm{~mm}$.

-to obtain high-quality air-droplet flow, the initial speed of the main droplets discharged from the periphery of the spray disc should be less than the air velocity and rotational frequency Pavlovskogo spray is recommended to be applied with in $\omega=60 \ldots 200 \mathrm{~s}^{-1}$.

\section{References}

1. Aliev B.G. Application of herbicides in the cotton-growing zone of Uzbekistan, p. 212, Tashkent, (1971) 
2. Balazh F. Increasing the efficiency of plant protection by new methods and machines, International agricultural. Journal, № 1, p. 102, (1984)

3. Bayramov K. Defoliation of cotton on different backgrounds of mineral nutrition, Cotton growing, № 7, pp. 24-26, (1980)

4. Veletsky I.N. Herbicide application technology, Agropromizdat, p. 175, (1989)

5. Baranovskiy A.S., Oil I.P., Timoshenko S.P. Investigation of the influence of the shape of the outlet section of the nozzle (fan) of the sprayer on the distribution of the liquid, Mechanization and electrification of agriculture, 60, pp. 10-12, Kiev, (1984)

6. Baranovsky A.S. Investigation of the process of spraying field crops with aerosols of pesticides and substantiation of the parameters of spraying organs, p. 195, Kiev, (1979)

7. Geisbüller I.M. Chemical method - present and future, Plant protection, № 4, pp. 4041 (1982)

8. Targ S.M. Theoretical Mechanics Course, p. 415Moscow, Science, (1986)

9. Korn G., Korn T. Handbook of mathematics for scientists and engineers, p. 720, Moscow, Science, (1990)

10. Lee A., Allaniyazov S., I. Khudaev T., Usmonov I., Gorlova. Modeling The Motion Of Alferia Seeds In The Sorting Device, International Jornal of Advanced and Technology, 29(7) pp. 3431-3435, (2020)

11. Lee A., Usmonov T., Norov B. Seiling and cleaning of channels, IOP Conference Series, Materials Science and Engineering 883 p. 012062, (2020), doi: 10.1088 / 1757-899X / 883/1/012062

12. Lee, A., Usmonov, T., Norov, B., Melikuziev, S. Advanced device for cleaning drain wells. IOP Conference Series: Materials Science and Engineering, 883 (1), p. 012181 , (2020), doi: 10.1088 / 1757-899X / 883/1/012181 Tashkent Institute of Irrigation and Agricultural Mechanization Engineers, Tashkent, Uzbekistan.

13. Donsky V.F., Nikitin N.V. Monodisperse rotating sprayers, Mechanization and electrification of agriculture, № 8, pp. 11-14 (1981)

14. Investigation of aerodynamic parameters of the working body of the cotton sprayer OBX-28, GSKB report on machines for cotton growing, p. 89 (1982)

15. B. Utepov. Justification of the basic parameters and operating modes of the pneumatic disc sprayer of the low-volume cotton sprayer, p.18, (1993)

16. Gushchin E.G. Investigation of the technological process of a small-volume cotton sprayer in order to optimize the design parameters of the jet-forming device of the fan sprayer, p. 204 Tajikistan, (1976)

17. Muratov, A., Muratov, O., Melikuziyev, S. Operational control of energy consumptions of reclamation machines IOP Conference Series, Earth and Environmental Science, 614 (1), (2020) doi: 10.1088/1755-1315/614/1/012042 